Pacific Journal of Mathematics

PLURISUBHARMONIC DEFINING FUNCTIONS 


\section{PLURISUBHARMONIC DEFINING FUNCTIONS}

\section{JOHN ERIK FORNAESS}

Let $\Omega$ be a bounded pseudoconvex open set in $n$-dimensional complex Euclidean space $C^{n}$ with a smooth $\left(\mathscr{C}^{\infty}\right)$ boundary. It has been known for some time that it is not always possible to choose a defining function $\rho$ which is plurisubharmonic in a neighborhood of $\bar{\Omega}$. We study here the question whether for every point $p \in \partial \Omega$, there exists an open neighborhood on which $\rho$ can be chosen to be plurisubharmonic. Our main conclusion is that this is not always the case.

1. Notation and results. In what follows, $\Omega$ will always be a bounded open set in $C^{n}$ with $\mathscr{C}^{\infty}$-boundary. This means that there exists a real-valued $\mathscr{C}^{\infty}$-function $\rho: C^{n} \rightarrow \boldsymbol{R}$ such that $\Omega=\{\rho<0\}$ and $d \rho \neq 0$ on $\partial \Omega$. Let $z=\left(z_{1}, z_{2}, \cdots, z_{n}\right), z_{j}=x_{j}+i y_{j}$, denote complex coordinates in $C^{n}$, and define

$$
\frac{\partial}{\partial z_{j}}=\frac{1}{2}\left(\frac{\partial}{\partial x_{j}}-i \frac{\partial}{\partial y_{j}}\right), \quad \frac{\partial}{\partial \bar{z}_{j}}=\frac{1}{2}\left(\frac{\partial}{\partial x_{j}}+i \frac{\partial}{\partial y_{j}}\right) .
$$

Definition 1. The set $\Omega$ is pseudoconvex if for every $p \in \partial \Omega$, we have

$$
\sum_{i, j=1}^{n} \frac{\partial^{2} \rho}{\partial z_{i} \partial \bar{z}_{j}}(p) t_{i} \bar{t}_{j} \geqq 0
$$

whenever

$$
t=\left(t_{1}, \cdots, t_{n}\right) \in C^{n}-(0) \text { and } \sum_{i=1}^{n} \frac{\partial \rho}{\partial z_{i}}(p) t_{i}=0 .
$$

If we have strict inequality in (1) for all $p \in \partial \Omega$, then $\Omega$ is said to be strongly pseudoconvex.

Definition 2. A real-valued $\mathscr{B}^{2}$-function, $u$, defined on an open set $V$ in $C^{n}$ is plurisubharmonic if

$$
\sum_{i, j=1}^{n} \frac{\partial^{2} u}{\partial z_{i} \partial \bar{z}_{j}}(p) t_{i} \bar{t}_{j} \geqq 0
$$

whenever $p \in V$ and $t=\left(t_{1}, \cdots, t_{n}\right) \in C^{n}-(0)$.

If we have strict inequality for all $p \in V$, then $u$ is strictly plurisubharmonic. 
The following results are known:

THEOREM 3 [2]. If $\Omega$ is strongly pseudoconvex, then $\rho$ may be chosen to be strictly plurisubharmonic in some neighborhood of $\bar{\Omega}$.

The next example shows that the theorem fails in general if we drop the hypothesis of strong pseudoconvexity.

EXAmple 4 [1]. There exists a bounded pseudoconvex domain $\Omega$ in $C^{2}$, with $\mathscr{C}^{\infty}$-boundary, such that no $\left(\mathscr{C}^{2}\right)$ defining function $\rho$ exists with

$$
\sum_{i, j=1}^{n} \frac{\partial^{2} \rho}{\partial z_{i} \partial \bar{z}_{j}}(p) t_{i} \bar{t}_{j} \geqq 0
$$

whenever

$$
p \in \partial \Omega \text { and } t=\left(t_{1}, \cdots, t_{n}\right) \in C^{n} .
$$

There exists an example, similar to the one above, which has a real analytic boundary.

EXAMPLE 5. Let

$$
\begin{aligned}
\Omega & =\Omega_{K}=\left\{\left(z_{1}, z_{2}\right) \in(C-(0)) \times C ; \sigma\right. \\
& \left.=\left|z_{2}+e^{i \ln z_{1} \bar{z}_{1}}\right|^{2}-1+K\left(\ln z_{1} \bar{z}_{1}\right)^{4}<0\right\} .
\end{aligned}
$$

Then, if, $K>1$ is sufficiently large, $\Omega$ is a bounded pseudoconvex domain in $C^{2}$ with smooth real analytic boundary, such that no $\mathscr{C}^{2}$ defining function, $\rho$, exists such that

$$
\sum_{i, j=1}^{2} \frac{\partial^{2} \rho}{\partial z_{i} \partial \bar{z}_{j}}(p) t_{i} \bar{t}_{j} \geqq 0
$$

whenever $p \in \partial \Omega$ and $\left(t_{1}, t_{2}\right) \in C^{2}$.

The details will be given in the next section.

EXAMPle 6. There exists a bounded pseudoconvex domain $\Omega$ in $C^{3}$, with $\mathscr{C}^{\infty}$-boundary, and a point $p \in \partial \Omega$ such that whenever $\rho$ is a $\sigma^{\prime 2}$ defining function for $\Omega$,

$$
\sum_{i, j=1}^{3} \frac{\partial^{2} \rho}{\partial z_{i} \partial \bar{z}_{j}}(q) t_{i} \bar{t}_{j}<0
$$

for some $\left(t_{1}, \cdots, t_{n}\right)$ and $q \in \partial \Omega$ arbitrarily close to $p$.

This example shows that one does not have plurisubharmonic 
defining functions for pseudoconvex domains, even locally, in general.

\section{Examples.}

Example 5. Clearly, $\Omega$ is bounded in $(\boldsymbol{C}-(0)) \times \boldsymbol{C}$. If $\partial \sigma / \partial z_{2}=$ 0 , then $z_{2}=-e^{i \ln z_{1} \bar{z}_{1}}$. Hence, if $d \sigma=0$, then $0=z_{1} \partial \sigma / \partial z_{1}=4 K\left(\ln z_{1} \bar{z}_{1}\right)^{3}$. This implies that $\left|z_{1}\right|=1$ and $z_{2}=-1$. At such points, $\sigma\left(z_{1}, z_{2}\right)=$ -1 , so $d \sigma \neq 0$ on $\partial \Omega$.

To show that $\Omega$ is pseudoconvex, we compute the Leviform

$$
\begin{aligned}
\mathscr{L}= & \frac{\partial^{2} \sigma}{\partial z_{1} \partial \bar{z}_{1}}\left|\frac{\partial \sigma}{\partial z_{2}}\right|^{2}-\frac{\partial^{2} \sigma}{\partial z_{1} \partial \bar{z}_{2}} \frac{\partial \sigma}{\partial z_{2}} \frac{\partial \sigma}{\partial \bar{z}_{1}}-\frac{\partial^{2} \sigma}{\partial \bar{z}_{1} \partial z_{2}} \cdot \frac{\partial \sigma}{\partial z_{1}} \cdot \frac{\partial \sigma}{\partial \bar{z}_{2}} \\
& +\frac{\partial^{2} \sigma}{\partial z_{2} \partial \bar{z}_{2}} \cdot\left|\frac{\partial \sigma}{\partial z_{1}}\right|^{2}
\end{aligned}
$$

to obtain

$$
\begin{aligned}
\mathscr{L}= & \frac{z_{2} \bar{z}_{2}+K\left(\ln z_{1} \bar{z}_{1}\right)^{4}+12 K\left(\ln z_{1} \bar{z}_{1}\right)^{2}}{z_{1} \bar{z}_{1}} \cdot\left|z_{2}+e^{i \ln z_{1} \bar{z}_{1}}\right|^{2} \\
& +4 K \frac{\left(\ln z_{1} \bar{z}_{1}\right)^{3}}{z_{1} \bar{z}_{1}}\left(i \bar{z}_{2} e^{i \ln z_{1} \bar{z}_{1}}-i z_{2} e^{-i \ln z_{1} \bar{z}_{1}}\right)+16 K^{2} \frac{\left(\ln z_{1} \bar{z}_{1}\right)^{6}}{z_{1} \bar{z}_{1}}
\end{aligned}
$$

on $\partial \Omega$.

If $\left|z_{2}+e^{i \mathrm{inz} z_{1} \overline{z_{1}}}\right| \geqq 1 / 2$, we have

$$
\mathscr{L} \geqq 3 K\left(\ln z_{1} \bar{z}_{1}\right)^{2} / z_{1} \bar{z}_{1}-16 K\left|\ln z_{1} \bar{z}_{1}\right|^{3} / z_{1} \bar{z}_{1},
$$

since $\left|z_{2}\right| \leqq 2$ on $\partial \Omega$. If $K$ is sufficiently large, then $\left|\ln z_{1} \bar{z}_{1}\right|<3 / 16$ on $\partial \Omega$ and hence $\mathscr{L} \geqq 0$.

Consider next a boundary point where $\left|z_{2}+e^{i \ln z_{1} \bar{z}_{1}}\right|<1 / 2$. Then $K\left(\ln z_{1} \bar{z}_{1}\right)^{4} \geqq 3 / 4$, since $\sigma\left(z_{1}, z_{2}\right)=0$. Hence

$$
\begin{aligned}
\mathscr{L} & \geqq-16 K\left|\ln z_{1} \bar{z}_{1}\right|{ }^{3} / z_{1} \bar{z}_{1}+16 K^{2}\left(\ln z_{1} \bar{z}_{1}\right)^{6} / z_{1} \bar{z}_{1} \\
& =16 K\left|\ln z_{1} \bar{z}_{1}\right|^{3} / z_{1} \bar{z}_{1}\left(-1+K\left(\ln z_{1} \bar{z}_{1}\right)^{4} /\left|\ln z_{1} \bar{z}_{1}\right|\right)
\end{aligned}
$$

which is nonnegative if $K$ is sufficiently large.

Assume next that $\rho$ is a $\mathscr{C}^{2}$ defining function for $\Omega$ such that

$$
\sum_{i, j=1}^{2} \frac{\partial^{2} \rho}{\partial z_{i} \partial \bar{z}_{j}}(p) t_{i} \bar{t}_{j} \geqq 0
$$

whenever $p \in \partial \Omega$ and $\left(t_{1}, t_{2}\right) \in C^{2}$. In particular, $\rho=h \sigma$ for some $\mathscr{C}^{1-}$ function $h>0$. We observe that $\partial^{2} \rho / \partial z_{1} \partial \bar{z}_{1}\left(z_{1}, z_{2}\right)=0$ whenever $\left|z_{1}\right|=1$ and $z_{2}=0$. (All such points are in $\partial \Omega$.) Therefore, $\partial^{2} \rho / \partial \bar{z}_{1} \partial z_{2}\left(z_{1}, z_{2}\right)=0$ at these points also. Hence 


$$
\left(\frac{\partial h}{\partial \bar{z}_{1}} \frac{\partial \sigma}{\partial z_{2}}+h \frac{\partial^{2} \sigma}{\partial \bar{z}_{1} \partial z_{2}}\right)\left(e^{i \theta}, 0\right) \equiv 0
$$

and so

$$
\frac{\partial}{\partial \bar{z}_{1}}\left(h e^{i \ln \bar{z}_{1} \bar{z}_{1}}\right)\left(e^{i \theta}, 0\right) \equiv 0 .
$$

Multiplying with $e^{i \log z_{1}}$ we get that

$$
\frac{\partial}{\partial \bar{z}_{1}}\left(h e^{-2 \operatorname{Arg} z_{1}}\right)\left(e^{i \theta}, 0\right) \equiv 0
$$

which implies that $h\left(e^{i \theta}, 0\right)=c e^{2 \theta}$ for some constant $c>0$. This is of course impossible.

In the next example, we localize the above idea suitably.

EXAMPLE 6. Let us use coordinates $\left(w, z_{1}, z_{2}\right)$ in $C^{3}$ with $w=$ $\eta+i \zeta$ and $z_{j}=x_{j}+i y_{j}, j=1,2$. We pick a $\mathscr{C}^{\infty}$, convex function $\chi_{1}(t): R \rightarrow R$ such that $\chi_{1}(t)=0$ when $t \leqq 1$ and $\chi_{1}(t)>0$ when $t>0$. Define $\sigma_{1}: C^{3} \rightarrow R$ by

$$
\sigma_{1}=\eta+\eta^{2}+K \zeta^{2}+K\left(y_{1}^{2}+y_{2}^{2}\right)^{2}+\left(y_{1}^{2}+y_{2}^{2}\right) \zeta^{2}+\chi_{1}\left(x_{1}^{2}+x_{2}^{2}\right),
$$

and let $\Omega_{1}=\left\{\sigma_{1}<0\right\}$. Here $K \gg 1$ is a constant which will be chosen later.

Lemma 7. The set $\Omega_{1}$ is bounded and pseudoconvex with $\mathscr{C}^{\infty}$ boundary for all $K$ sufficiently large.

Proof. Computation shows that $d \sigma_{1}=0$ only at points $\left(-1 / 2, x_{1}, x_{2}\right)$ with $x_{1}^{2}+x_{2}^{2} \leqq 1$. Since $\sigma_{1}=-1 / 4$ at these points, it follows that $d \sigma_{1} \neq 0$ on $\partial \Omega_{1}$. Further computation shows that $\sigma_{1}$ is plurisubharmonic in a neighborhood of $\bar{\Omega}_{1}$ if $K$ is sufficiently large.

In the following $K$, sufficiently large, is fixed.

The next step is to make an infinite number of perturbations of the boundary of $\Omega_{1}$. Let $p_{j}=\left(0,1 / 2^{j}, 0\right), j=1,2, \cdots$ and let $\boldsymbol{B}\left(p_{j}, r\right)=\left\{\left(w, z_{1}, z_{2}\right) ;\left(|w|^{2}+\left|z_{1}-1 / 2^{j}\right|^{2}+\left|z_{2}\right|^{2}\right)^{1 / 2}<r\right\}$ be the ball centered at $p_{j}$ of radius $r$. Choose functions $\chi^{(j)} \in \mathscr{C}_{0}^{\infty}\left(B\left(p_{j}, 1 / 2^{j+2}\right)\right)$ with $\chi^{(j)} \equiv 1$ on $B\left(p_{j}, 1 / 2^{j+3}\right)$ and $\chi^{(j)} \geqq 0, j=1,2, \ldots$. Observe that supp $\chi^{(i)} \cap \operatorname{supp} \chi^{(j)}=\varnothing$ whenever $i \neq j$. We may arrange that $\left|d \chi^{(j)}\right|^{2} \leqq C_{j} \chi^{(j)}$ and $\left|\partial \chi^{(j)} / \partial y_{k}\right| \leqq C_{j}\left|y_{k}\right|$ for suitable $C_{1}, C_{2}, \cdots$, and $k=1,2$. Let $\varepsilon=\left\{\varepsilon_{j}\right\}_{j=1}^{\infty}$ denote a rapidly decreasing sequence, $\varepsilon_{1}>$ $\varepsilon_{2}>\cdots>0$ and define

$$
\sigma_{2}=\sigma_{1}+\sum_{j=1}^{\infty} \varepsilon_{j} \chi^{(j)} \cdot\left(y_{1}^{2}+y_{2}^{2}\right) \cdot x_{2}^{2} .
$$


Clearly $\sigma_{2}$ is a $\mathscr{C}^{\infty}$-function, and if $\Omega_{2}=\left\{\sigma_{2}<0\right\}$, then $d \sigma_{2} \neq 0$ on $\partial \Omega_{2}$ and $\Omega_{2}$ is a bounded domain which is pseudoconvex at every point in $\partial \Omega_{2}-\bigcup_{j} B\left(p_{j}, 1 / 2^{j+2}\right)$.

Lemma 8. The set $\Omega_{2}$ is pseudoconvex if $\varepsilon$ decreases sufficiently fast.

Proof. Fix a $j \geqq 1$. It suffices to show that $\sigma_{1}+\varepsilon_{j} \chi^{(j)} \cdot\left(y_{1}^{2}+y_{2}^{2}\right) x_{2}^{2}$ is plurisubharmonic in $\boldsymbol{B}\left(p_{j}, 1 / 2^{j+2}\right)$ for all small enough $\varepsilon_{j}>0$. This is checked by a direct computation.

We fix a sequence $\left\{\varepsilon_{j}\right\}$ decreasing sufficiently fast.

To complete the construction of the example, we will perturbe $\sigma_{2}$ inside each $B\left(p_{j}, 1 / 2^{j+3}\right)$. More precisely, let $\chi_{(j)} \in \mathscr{C}_{0}^{\infty}\left(B\left(p_{j}, 1 / 2^{j+3}\right)\right)$ with

$$
\int_{R}\left(\frac{\partial \chi_{(j)}}{\partial x_{1}}+\chi_{(j)}\right)\left(0, x_{1}, 0\right) d x_{1} \neq 0
$$

for each $j, \chi_{(j)} \geqq 0$. We may assume that $\left|\partial \chi_{(j)} / \partial \eta\right|,\left|\partial \chi_{(j)} / \partial \zeta\right|,\left|\partial \chi_{(j)} / \partial y_{k}\right|$, $\left|\partial \chi_{(j)} / \partial x_{2}\right| \leqq C_{j}\left(|\eta|+|\zeta|+\left|x_{2}\right|+\left|y_{1}\right|+\left|y_{2}\right|\right), k=1,2, C_{j}$ some constant.

If $\delta=\left\{\delta_{j}\right\}_{j=j_{0}}^{\infty}, \delta_{j_{0}}>\delta_{j_{0}+1}>\cdots>0$ is any sufficiently rapidly decreasing sequence,

$$
\sigma=\sigma_{2}+\sum_{j=j_{0}}^{\infty} \delta_{j} \chi_{(j)} \cdot\left(\eta+\zeta y_{1}\right)
$$

is a $\mathscr{C}^{\infty}$-function and $d \sigma \neq 0$ on $\partial \Omega, \Omega=\{\sigma<0\}$. Moreover, $\Omega$ is a bounded domain which is pseudoconvex on $\partial \Omega-\cup \boldsymbol{B}\left(p_{j}, 1 / 2^{j+3}\right)$.

Lemma 9. The set $\Omega$ is pseudoconvex if $\delta$ decreases sufficiently fast, and $j_{0}$ is sufficiently large.

Proof. Fix a $j \gg 1$. It suffices to show that $\Omega$ is pseudoconvex at those boundary points which are in $B\left(p_{j}, 1 / 2^{j+3}\right)$ for all $\delta_{j}$ sufficiently small. In $\boldsymbol{B}\left(p_{j}, \mathbf{1} / 2^{j+3}\right), \sigma=\eta+\eta^{2}+K \zeta^{2}+K\left(y_{1}^{2}+y_{2}^{2}\right)^{2}+\left(y_{1}^{2}+y_{2}^{2}\right) \zeta^{2}+$ $\varepsilon_{j}\left(y_{1}^{2}+y_{2}^{2}\right) \cdot x_{2}^{2}+\delta_{j} \chi_{(j)} \cdot\left(\eta+\zeta y_{1}\right)$. Differentiating, we obtain:

$$
\begin{aligned}
\frac{\partial \sigma}{\partial w}= & \frac{1}{2}+\eta-i K \zeta-i \zeta\left(y_{1}^{2}+y_{2}^{2}\right)+\delta_{j} \frac{\delta \chi_{(j)}}{\delta w} \cdot\left(\eta+\zeta y_{1}\right) \\
& +\frac{1}{2} \delta_{j} \chi_{(j)}-\frac{i}{2} \delta_{j} \chi_{(j)} y_{1}, \\
\frac{\partial \sigma}{\partial z_{1}}= & -2 i K\left(y_{1}^{3}+y_{1} y_{2}^{2}\right)-i y_{1} \zeta^{2}-i \varepsilon_{j} y_{1} x_{2}^{2} \\
& +\delta_{j} \frac{\partial \chi_{(j)}}{\partial z_{1}} \cdot\left(\eta+\zeta y_{1}\right)-\frac{i}{2} \delta_{j} \chi_{(j)} \cdot \zeta,
\end{aligned}
$$




$$
\begin{aligned}
\frac{\partial \sigma}{\partial z_{2}}= & -2 i K\left(y_{1}^{2} y_{2}+y_{2}^{3}\right)-i y_{2} \zeta^{2}-i \varepsilon_{j} y_{2} x_{2}^{2}+\varepsilon_{j}\left(y_{1}^{2}+y_{2}^{2}\right) x_{2} \\
& +\delta_{j} \frac{\partial \chi_{(j)}}{\partial z_{2}} \cdot\left(\eta+\zeta y_{1}\right) \\
\frac{\partial^{2} \sigma}{\partial w \partial \bar{w}}= & \frac{1}{2}+\frac{K}{2}+\frac{1}{2}\left(y_{1}^{2}+y_{2}^{2}\right)+\delta_{j} \frac{\partial^{2} \chi_{(j)}}{\partial w \partial \bar{w}} \cdot\left(\eta+\zeta y_{1}\right) \\
& +\frac{1}{2} \delta_{j} \frac{\partial \chi_{(j)}}{\partial w}+\frac{i}{2} \delta_{j} \frac{\partial \chi_{(j)}}{\partial w} \cdot y_{1}+\frac{1}{2} \delta_{j} \frac{\partial \chi_{(j)}}{\partial \bar{w}} \\
& -\frac{i}{2} \delta_{j} \frac{\partial \chi_{(j)}}{\partial \bar{w}_{1}} \cdot y_{1}, \\
\frac{\partial^{2} \sigma}{\partial w \partial \bar{z}_{1}}= & \zeta y_{1}+\delta_{j} \frac{\partial^{2} \chi_{(j)}}{\partial w \partial \bar{z}_{1}} \cdot\left(\eta+\zeta y_{1}\right)+\frac{i}{2} \delta_{j} \frac{\partial \chi_{(j)}}{\partial w} \cdot \zeta \\
& +\frac{1}{2} \delta_{j} \frac{\partial \chi_{(j)}}{\partial \bar{z}_{1}}-\frac{i}{2} \delta_{j} \frac{\partial \chi_{(j)}}{\partial \bar{z}_{1}} y_{1}+\frac{1}{4} \delta_{j} \chi_{(j)}, \\
\frac{\partial^{2} \sigma}{\partial w \partial \bar{z}_{2}}= & \zeta y_{2}+\delta_{j} \frac{\partial^{2} \chi_{(j)}}{\partial w \partial \bar{z}_{2}} \cdot\left(\eta+\zeta y_{1}\right)+\frac{1}{2} \delta_{j} \frac{\partial \chi_{(j)}}{\partial \bar{z}_{2}}-\frac{i}{2} \delta_{j} \frac{\partial \chi_{(j)}}{\partial \bar{z}_{2}} \cdot y_{1}, \\
\frac{\partial^{2} \sigma}{\partial z_{1} \partial \bar{z}_{1}}= & 3 K y_{1}^{2}+K y_{2}^{2}+\frac{1}{2} \zeta^{2}+\frac{1}{2} \varepsilon_{j} x_{2}^{2}+\delta_{j} \frac{\partial^{2} \chi_{(j)}}{\partial z_{1} \partial \bar{z}_{1}} \cdot\left(\eta+\zeta y_{1}\right) \\
& +\frac{i}{2} \delta_{j} \frac{\partial \chi_{(j)}}{\partial z_{1}} \cdot \zeta-\frac{i}{2} \delta_{j} \frac{\partial \chi_{(j)}}{\partial \bar{z}_{1}} \cdot \zeta, \\
\frac{\partial^{2} \sigma}{\partial z_{1} \partial \bar{z}_{2}}= & 2 K y_{1} y_{2}-i \varepsilon_{j} y_{1} x_{2}+\delta_{j} \frac{\partial^{2} \chi_{(j)}}{\partial z_{1} \partial \bar{z}_{2}} \cdot\left(\eta+\zeta y_{1}\right)-\frac{i}{2} \delta_{j} \frac{\partial \chi_{(j)}}{\partial \bar{z}_{2}} \cdot \zeta
\end{aligned}
$$

and

$$
\begin{aligned}
\frac{\partial^{2} \sigma}{\partial z_{2} \partial \bar{z}_{2}}= & K y_{1}^{2}+3 K y_{2}^{2}+\frac{1}{2} \zeta^{2}+\frac{\varepsilon_{j}}{2} x_{2}^{2}-i \varepsilon_{j} x_{2} y_{2}+i \varepsilon_{j} y_{2} x_{2} \\
& +\frac{1}{2} \varepsilon_{j}\left(y_{1}^{2}+y_{2}^{2}\right)+\delta_{j} \frac{\partial^{2} \chi_{(j)}}{\partial z_{2} \partial \bar{z}_{2}} \cdot\left(\eta+\zeta y_{1}\right) .
\end{aligned}
$$

Observe that $\eta=0\left(\zeta^{2}+y_{1}^{2}+y_{2}^{2}\right)$ on $\partial \Omega \cap \boldsymbol{B}\left(p_{j}, 1 / 2^{j+3}\right)$. Hence there is a $D_{j} \gg 1$ such that for all sufficiently small $\delta_{j}>0, \partial^{2} \sigma / \partial w \partial \bar{w} \geqq K / 2$,

$$
\begin{aligned}
& \left|\frac{\partial^{2} \sigma}{\partial w \partial \bar{z}_{1}}-\zeta y_{1}-\frac{1}{4} \delta_{j} \frac{\partial \chi_{(j)}}{\partial x_{1}}-\frac{1}{4} \delta_{j} \chi_{(j)}\right| \leqq D_{j} \delta_{j}\left\|\left(w, i y_{1}, z_{2}\right)\right\|, \\
& \left|\frac{\partial^{2} \sigma}{\partial w \partial \bar{z}_{2}}-\zeta y_{2}\right| \leqq D_{j} \delta_{j}\left\|\left(w, i y_{1}, z_{2}\right)\right\| \\
& \frac{\partial^{2} \sigma}{\partial z_{1} \partial \bar{z}_{1}} \geqq(3 K-1) y_{1}^{2}+(K-1) y_{2}^{2}+\frac{1}{4} \zeta^{2}+\frac{1}{4} \varepsilon_{j} x_{2}^{2}, \\
& \left|\frac{\partial^{2} \sigma}{\partial z_{1} \partial \bar{z}_{2}}-2 K y_{1} y_{2}+i \varepsilon_{j} y_{1} x_{2}\right| \leqq D_{j} \delta_{j}\left\|\left(w, i y_{1}, z_{2}\right)\right\|^{2}
\end{aligned}
$$

and 


$$
\frac{\partial^{2} \sigma}{\partial z_{2} \partial \bar{z}_{2}} \geqq K y_{1}^{2}+3 K y_{2}^{2}+\frac{1}{4} \zeta^{2}+\frac{\varepsilon_{j}}{4} x_{2}^{2}
$$

We compute the Leviform,

$$
\begin{aligned}
\mathscr{L}_{\sigma}=\sigma_{w \bar{u}} t_{0} \bar{t}_{0} & +2 \operatorname{Re} \sigma_{w \bar{z}_{1}} t_{0} \bar{t}_{1}+2 \operatorname{Re} \sigma_{w \overline{z_{2}}} t_{0} \bar{t}_{2} \\
& +\sigma_{z_{1} \bar{z}_{1}} t_{1} \bar{t}_{1}+2 \operatorname{Re} \sigma_{z_{1} \bar{z}_{2}} t_{1} \bar{t}_{2}+\sigma_{z_{2} \bar{z}_{2}} t_{2} \bar{t}_{2}
\end{aligned}
$$

for vectors $\left(t_{0}, t_{1}, t_{2}\right)$ such that

$$
t_{0}=\left(-1 / \sigma_{w}\right) \cdot\left(\sigma_{z_{1}} t_{1}+\sigma_{z_{2}} t_{2}\right) .
$$

Using the above estimates, we obtain

$$
\begin{aligned}
\mathscr{C}_{\sigma} \geqq & \left((3 K-2) y_{1}^{2}+(K-2) y_{2}^{2}+\frac{1}{8} \zeta^{2}+\frac{1}{8} \varepsilon_{j} x_{2}^{2}\right) t_{1} \bar{t}_{1} \\
+ & \left((K-2) y_{1}^{2}+(3 K-2) y_{2}^{2}+\frac{1}{8} \zeta^{2}+\frac{\varepsilon_{j}}{8} x_{2}^{2}\right) t_{2} \bar{t}_{2} \\
+ & 2 \operatorname{Re}\left(2 K y_{1} y_{2}-i \varepsilon_{j} y_{1} x_{2}\right) t_{1} \bar{t}_{2} \\
+ & 2 \operatorname{Re}\left(\frac{1}{4} \delta_{j} \frac{\partial \chi_{(j)}}{\partial x_{1}}+\frac{1}{4} \delta_{j} \chi_{(j)}\right) \cdot\left[\left(\frac{-1}{\frac{1}{2}+\frac{1}{2} \delta_{j} \chi_{(j)}}\right) \cdot \frac{-i}{2}\right. \\
& \left.\times \delta_{j} \chi_{(j)} \zeta t_{1}\right] \bar{t}_{1}
\end{aligned}
$$

which clearly is nonnegative.

Assume that there exists a $\mathscr{C}^{2}$-function $\rho: \boldsymbol{C}^{3} \rightarrow \boldsymbol{R}$, such that $\Omega=\{\rho<0\}$ and $d \rho \neq 0$ on $\partial \Omega$, with a nonnegative complex Hessian on some neighborhood $U$ of 0 in $\partial \Omega$.

Let $\gamma_{i}, i=1,2,3,4$, be straight lines in the $\left(x_{1}, x_{2}\right)$-plane,

$$
\begin{aligned}
& \gamma_{1} \text { goes from }\left(\frac{1}{2^{j}}-\frac{1}{2^{j+2}}, 0\right) \text { to }\left(\frac{1}{2^{j}}+\frac{1}{2^{j+2}}, 0\right) \\
& \gamma_{2} \text { goes from }\left(\frac{1}{2^{j}}+\frac{1}{2^{j+2}}, 0\right) \text { to }\left(\frac{1}{2^{j}}+\frac{1}{2^{j+2}}, \frac{1}{2^{j+2}}\right), \\
& \gamma_{3} \text { goes from }\left(\frac{1}{2^{j}}+\frac{1}{2^{j+2}}, \frac{1}{2^{j+2}}\right) \text { to }\left(\frac{1}{2^{j}}-\frac{1}{2^{j+2}}, \frac{1}{2^{j+2}}\right) \text { and } \\
& \gamma_{4} \text { goes from }\left(\frac{1}{2^{j}}-\frac{1}{2^{j+2}}, \frac{1}{2^{j+2}}\right) \text { to }\left(\frac{1}{2^{j}}-\frac{1}{2^{j+2}}, 0\right) .
\end{aligned}
$$

We fix $j$ so large that each $\gamma_{i} \subset U$. The function $\rho=\sigma h$ for some $\mathscr{C}^{1}$-function $h>0$. 
We will show that $\int_{r_{1}} d(\ln h) \neq 0$ for all small enough $\hat{o}_{j}>0$, while

$$
\int_{\gamma_{i}} d(\ln h)=0, i=2,3,4 \text {. }
$$

First consider the curves $\gamma_{2}$ and $\gamma_{4}$. There $\rho=\left(\eta+\eta^{2}+K \zeta^{2}+\right.$ $\left.K\left(y_{1}^{2}+y_{2}^{2}\right)^{2}+\left(y_{1}^{2}+y_{2}^{2}\right) \zeta^{2}\right) h$ from which it follows that $\partial^{2} \rho / \partial z_{2} \partial \bar{z}_{2} \equiv 0$ on $\gamma_{2} \cup \gamma_{4}$. Hence $\partial^{2} \rho / \partial w \partial \bar{z}_{2} \equiv 0$ on $\gamma_{2} \cup \gamma_{4}$ as well. This reduces to the equation $\partial h / \partial \bar{z}_{2}=0$ from which it follows that $\int_{i_{i}} d(\ln h)=0, i=2,4$. Similarly $\int_{r_{3}} d(\ln h)=0$.

Finally, consider the curve $\gamma_{1}$. Here $\sigma=\eta+\eta^{2}+K \zeta^{2}+K\left(y_{1}^{2}+y_{2}^{2}\right)^{2}+$ $\left(y_{1}^{2}+y_{2}^{2}\right) \zeta^{2}+\varepsilon_{j} \chi^{(j)} \cdot\left(y_{1}^{2}+y_{2}^{2}\right) \cdot x_{2}^{2}+\delta_{j} \chi_{(j)} \cdot\left(\eta+\zeta y_{1}\right)$. Clearly $\partial^{2} \rho / \partial z_{1} \partial \bar{z}_{1} \equiv 0$ on $\gamma_{1}$ and hence $\partial^{2} \rho / \partial w \partial \bar{z}_{1} \equiv 0$ there also. This reduces to the equation

$$
\partial^{2} \sigma / \partial w \partial \bar{z}_{1} \cdot h+\partial \sigma / \partial w \cdot \partial h / \partial \bar{z}_{1} \equiv 0 \quad \text { on } \quad \gamma_{1} \cdot
$$

Hence

$$
\frac{\partial}{\partial x_{1}}(\ln h)=\left(-\delta_{j}\right)\left(\partial \chi_{(j)} / \partial x_{1}+\chi_{(j)}\right) /\left(1+\delta_{j} \chi_{(j)}\right) .
$$

Since we choose $\chi_{(j)}$ such that

$$
\int_{R}\left(\frac{\partial \chi_{(j)}}{\partial x_{1}}+\chi_{(j)}\right)\left(0, x_{1}, 0\right) d x_{1} \neq 0
$$

it follows that $\int_{r_{1}} d(\ln h) \neq 0$ for all small enough $\delta_{j}>0$.

So $\int_{r_{1}+\cdots+r_{4}} d(\ln h) \neq 0$, which contradicts the assumption that $h$ was well ${ }^{\text {defined. }}$

\section{REFERENCES}

1. K. Diederich and J. E. Fornaess, Pseudoconvex domains: An example with nontrivial Nebenhülle, Math, Ann., 225 (1977), 275-292.

2. J. Morrow and H. Rossi, Some theorems of algebraicity for complex spaces, J. Math. Soc. Japan, 27 (1975), 167-183.

Received May 12, 1978.

Princeton University

PRINCETON, NJ 08544 


\section{PACIFIC JOURNAL OF MATHEMATICS}

\section{EDITORS}

RICHARD ARENS (Managing Editor)

University of California

Los Angeles, CA 90024

Charles W. Curtis

University of Oregon

Eugene, OR 97403

C. C. MOORE

University of California

Berkeley, CA 94720
J. DUGUNDJI

Department of Mathematics

University of Southern California

Los Angeles, CA 90007

R. FINN and J. MILGRAM

Stanford University

Stanford, CA 94305

\section{ASSOCIATE EDITORS}

\section{E. F. BeCKENBACH}

B. H. NeumanN

F. WOLF

K. YoSHIDA

\section{SUPPORTING INSTITUTIONS}

\author{
UNIVERSITY OF SOUTHERN CALIFORNIA \\ STANFORD UNIVERSITY \\ UNIVERSITY OF HAWAII \\ UNIVERSITY OF TOKYO \\ UNIVERSITY OF UTAH \\ WASHINGTON STATE UNIVERSITY \\ UNIVERSITY OF WASHINGTON
}

The Supporting Institutions listed above contribute to the cost of publication of this Journal, but they are not owners or publishers and have no responsibility for its content or policies.

Mathematical papers intended for publication in the Pacific Journal of Mathematics should be in typed form or offset-reproduced, (not dittoed), double spaced with large margins. Please do not use built up fractions in the text of the manuscript. However, you may use them in the displayed equations. Underline Greek letters in red, German in green, and script in blue. The first paragraph or two must be capable of being used separately as a synopsis of the entire paper. Items of the bibliography should not be cited there unless absolutely necessary, in which case they must be identified by author and journal, rather than by item number. Manuscripts, in triplicate, may be sent to any one of the editors. Please classify according to the scheme of Math. Reviews, Index to Vol. 39. All other communications should be addressed to the managing editor, or Elaine Barth, University of California, Los Angeles, California, 90024.

50 reprints to each author are provided free for each article, only if page charges have been substantially paid. Additional copies may be obtained at cost in multiples of 50 .

The Pacific Journal of Mathematics is issued monthly as of January 1966. Regular subscription rate: $\$ 72.00$ a year (6 Vols., 12 issues). Special rate: $\$ 36.00$ a year to individual members of supporting institutions.

Subscriptions, orders for numbers issued in the last three calendar years, and changes of address should be sent to Pacific Journal of Mathematics, P.O. Box 969, Carmel Valley, CA 93924, U.S.A. Older back numbers obtainable from Kraus Periodicals Co., Route 100, Millwood, NY 10546.

PUBLISHED BY PACIFIC JOURNAL OF MATHEMATICS, A NON-PROFIT CORPORATION

Printed at Kokusai Bunken Insatsusha (International Academic Printing Co., Ltd.). 8-8, 3-chome, Takadanobaba, Shinjuku-ku, Tokyo 160, Japan. 


\section{Pacific Journal of Mathematics}

\section{Vol. 80, No. $2 \quad$ October, 1979}

K. Adachi, On the multiplicative Cousin problems for $N^{p}(D) \ldots \ldots \ldots \ldots 297$

Howard Banilower, Isomorphisms and simultaneous extensions in $C(S) \ldots 305$

B. R. Bhonsle and R. A. Prabhu, An inversion formula for a distributional

finite-Hankel-Laplace transformation ................... 313

Douglas S. Bridges, Connectivity properties of metric spaces.......... 325

John Patton Burgess, A selection theorem for group actions ........... 333

Carl Claudius Cowen, Commutants and the operator equations

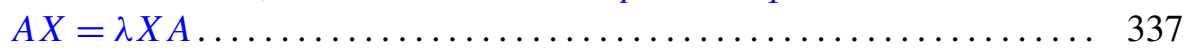

Thomas Curtis Craven, Characterizing reduced Witt rings. II .......... 341

J. Csima, Embedding partial idempotent d-ary quasigroups ............ 351

Sheldon Davis, A cushioning-type weak covering property ............ 359

Micheal Neal Dyer, Nonminimal roots in homotopy trees ............. 371

John Erik Fornaess, Plurisubharmonic defining functions ........... 381

John Fuelberth and James J. Kuzmanovich, On the structure of finitely

generated splitting rings .......................... 389

Irving Leonard Glicksberg, Boundary continuity of some holomorphic

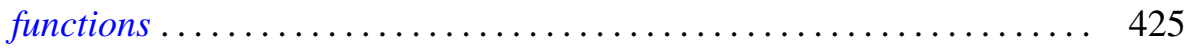

Frank Harary and Robert William Robinson, Generalized Ramsey theory.

IX. Isomorphic factorizations. IV. Isomorphic Ramsey numbers .......

Frank Harary and Allen John Carl Schwenk, The spectral approach to determining the number of walks in a graph...........

David Kent Harrison, Double coset and orbit spaces ..... . .

Shiro Ishikawa, Common fixed points and iteration of commuting

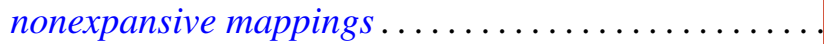

Philip G. Laird, On characterizations of exponential polynomials ........ 503

Y. C. Lee, A Witt's theorem for unimodular lattices ...........

Teck Cheong Lim, On common fixed point sets of commutative

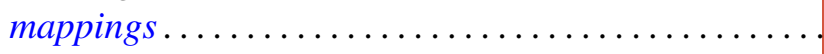

R. S. Pathak, On the Meijer transform of generalized functions ...

T. S. Ravisankar and U. S. Shukla, Structure of $\Gamma$-rings . . .

Olaf von Grudzinski, Examples of solvable and nonsolvable convolution

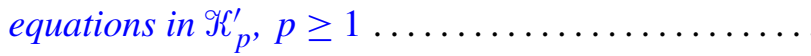

